\title{
Comments on the 'polynomial formulation and heuristic-based approach for the k-travelling repairman problem'
}

\author{
İmdat Kara
}

Department of Industrial Engineering, Baskent University Faculty of Engineering, Baglica Campus Ankara, Turkey

Email: ikara@baskent.edu.tr

\author{
Bahar Y. Kara* \\ Department of Industrial Engineering, \\ Bilkent University Faculty of Engineering, \\ Bilkent Campus Ankara, Turkey \\ Email: bkara@bilkent.edu.tr \\ *Corresponding author
}

\begin{abstract}
The paper 'polynomial formulation and heuristic-based approach for the k-travelling repairman problem' claims to present the first polynomial formulation for the k-travelling repairman problem (k-TRP). We first make some corrections on this formulation and we show that the first polynomial size formulation for k-TRP is the one proposed by Kara et al. (2008).
\end{abstract}

Keywords: repairman problem; k-travelling repairman problem; minimum latency problem; delivery man problem.

Reference to this paper should be made as follows: Kara, İ. and Kara, B.Y. (2019) 'Comments on the "polynomial formulation and heuristic-based approach for the k-travelling repairman problem', Int. J. Mathematics in Operational Research, Vol. 15, No. 1, pp.26-32.

Biographical notes: Imdat Kara is a Professor in the Department of Industrial Engineering at Baskent University. He received his BS from Mathematics and MSc from Operational Research and $\mathrm{PhD}$ on Quantitative Methods in Business. He works on mathematical programming in general and modelling of routing problems in particular. He has published many papers dealing with models and applications of routing problems in international journals. In addition, he wrote several operations research undergraduate/graduate text books all of which are in Turkish. His current research interests include distribution logistics, scheduling and bottleneck optimisation of routing problems.

Bahar Yetis Kara is a Professor in the Department of Industrial Engineering at Bilkent University. She works on operations research applications in logistics. She has published many papers dealing with models and applications of network optimisation problems. Her current research interests include distribution logistics, humanitarian logistics, hub location and hub network design, and hazardous material logistics. She is currently an Associate Editor of IIE Transactions. She is also one of the founders and so one of the members of the current executive board of the EURO Working Group on Humanitarian Operations (HOpe) 


\section{Introduction}

Let $G=(V, A)$ be a complete graph where $V$ is the set of nodes (vertices). A specific node of $\mathrm{V}$ is the depot (origin, home city) and the remaining nodes are customer nodes (clients). The set $A=\{(i, j): i, j \in V, i \neq j\}$ is an arc (or edge) set. With each $\operatorname{arc}(i, j)$ there is a travel time (or cost or distance). A traveller starting from the origin, visiting all the nodes of the graph and returning to origin perform a Hamiltonian tour. Latency of a customer on a tour (or path) is defined as time passed until the service of this client has been completed from the beginning of the journey. The minimum latency problem (MLP) is to find a tour starting at a depot and visiting all nodes in such a way that the total latency is minimised. This problem is also known as the delivery man problem (Fischetti et al., 1993) or the travelling repairman problem (TRP) (Tsitsiklis, 1992).

The k-travelling repairman problem (k-TRP) is a generalisation of the MLP and TRP that seeks k-tours, in such a way that, each repairman starts at the depot and each customer is visited by exactly one repairman while minimising total latency (Jothi and Raghavachari, 2007). For some cases of the k-TRP (e.g., delivering pizza), the distance (or travelling time) of the last arc on the tour may not be considered in the objective function. MLP and k-TRP can be considered as rather new problems to the routing area (see for ex. Angel-Bello et al., 2013; Önder et al., 2016; Nucamendi-Guillen et al., 2016; Flores-Garza et al., 2017; Kara et al., 2017).

Kara et al. (2008) defined a new variant of the vehicle routing problem as cumulative vehicle routing problem and propose a polynomial formulation with $O\left(n^{2}\right)$ binary variables and $O\left(n^{2}\right)$ constraints (where $\mathrm{n}$ is the number of the nodes of the underlying graph). They also show that, under special circumstances, their formulation reduces to a mathematical model for k-TRP. Later Ezzine and Elloumi (2012) presented a formulation for k-TRP claiming that they are the first to propose a mathematical programming for the problem.

In this note, we first make some corrections on Ezzine and Elloumi's (2012), formulation, abbreviated as EE. Then we list some comments on EE formulation and then show that, EE formulation is exactly same as Kara et al. (2008), (KKY), formulation. Thus, we conclude that Kara et al. (2008) formulation is actually the first paper where $\mathrm{k}$-TRP is formulated.

In Section 2, we give KKY and EE formulations, as they appeared where they are published. Some corrections and our comments are outlined in Section 3. We conclude with Section 4.

\section{KKY and EE formulations for k-TRP}

For both formulations, decision variables are defined as:

$$
x_{i j}= \begin{cases}1, & \text { if the } \operatorname{arc}(\mathrm{i}, \mathrm{j}) \text { is on the tour of a repairman } \\ 0, & \text { otherwise. }\end{cases}
$$

$y_{i j}$ is the number of remaining arcs on the tour (or path) from the node $i$ to the origin if a traveller goes from $i$ to $j$. 


\subsection{Kara et al. (2008) formulation}

Kara et al. (2008) define node set as $V=\{0,1,2, \ldots ., n\}$ where $\{0\}$ is the depot. The travel time from node $i$ to node $j$ shown as $d_{i j}$ (which also includes service time of the $j^{\text {th }}$ customer if exists). They consider the return time $d_{i o}$ to the depot, but mentioned that, for some cases of the k-TRP (e.g., delivering pizza), the travelling time (or distance) of the last arc on the tour may not be considered in the objective function.

We first present Kara et al. (2008) formulation, as follows:

$$
K K Y: \quad \operatorname{Min} \sum_{i=0}^{n} \sum_{j=0}^{n} d_{i j} y_{i j}
$$

Subject to

$$
\begin{array}{ll}
\sum_{i=1}^{n} x_{0 i}=m & \\
\sum_{i=1}^{n} x_{i 0}=m & \\
\sum_{i=0}^{n} x_{i j}=1, & j=1,2, \ldots, n \\
\sum_{j=0}^{n} x_{i j}=1, & i=1,2, \ldots, n \\
\sum_{\substack{j=0 \\
j \neq i}}^{n} y_{i j}-\sum_{\substack{j=0 \\
j \neq i}}^{n} y_{i j}=1, & i=1,2, \ldots, n \\
y_{i 0}=x_{i 0}, & i=1,2, \ldots, n \\
y_{i j} \leq(n-m) x_{i j}, & i \neq 0,(i, j) \in A \\
y_{i j} \geq 2 x_{i j}, & j \neq 0,(i, j) \in A \\
x_{i j} \in\{0,1\} & (i, j) \in A
\end{array}
$$

where $m$ is the number of the repairmen. Detailed explanations of the above formulations are given in Kara et al. (2008).

\subsection{Ezzine and Elloumi (2012) formulation}

Ezzine and Elloumi (2012) define the node set as $V=\{1,2, \ldots ., n\}$ where $\{1\}$ is the depot. $C_{i j}$ is defined as the travel cost on the arc $(i, j)$. Their formulation is given below as it originally appeared in this journal. 


$$
\text { EE : } \quad \operatorname{Min} \sum_{i=1}^{n} \sum_{j=1}^{n} c_{i j} x_{i j}
$$

Subject to

$$
\begin{aligned}
& \sum_{i=2}^{n} x_{1 i}=k \quad \forall(i=2, \ldots, n) \\
& \sum_{i=2}^{n} x_{i 1}=k \quad \forall(i=2, \ldots, n) \\
& \sum_{j=1}^{n} x_{i j}=1, \quad i=2, \ldots, n \\
& \sum_{i=1}^{n} x_{i j}=1, \quad j=2, \ldots, n \\
& x_{i j}+x_{j i}=1, \quad \forall(i, j=1,2, \ldots, n), i \neq j \\
& x_{i m}+x_{m j}+x_{j i} \leq 2, \quad \forall(i, j, m=1,2, \ldots, n), i \neq j \\
& x_{i j}+x_{j m}+x_{m i} \leq 2, \quad \forall(i, j, m=1,2, \ldots, n), i \neq j \\
& \sum_{j=1}^{n} y_{j i}-\sum_{j=1}^{n} y_{i j}=1, \quad \forall(i=2, \ldots, n), i \neq j \\
& y_{i 1}-x_{i 1}=0, \quad \forall(i=2, \ldots, n) \\
& y_{i j} \leq(n-k) x_{i j}, \quad \forall(i=2, \ldots, n)(j=1,2, \ldots, n), i \neq j \\
& y_{i j} \geq 2 x_{i j}, \quad \forall(j=2, \ldots, n)(i=1,2, \ldots, n), i \neq j \\
& y_{1 j} \leq n x_{1 j}, \quad \forall(j=2, \ldots, n) \\
& x_{i j} \in\{0,1\} \quad \forall(i, j=1,2, \ldots, n) \\
& y_{i j} \geq 0, y_{i j} \text { integer } \quad \forall(i, j=1,2, \ldots, n) \text {. }
\end{aligned}
$$

where $k$ is the number of the repairmen and $C_{i j}$ is defined as the cost of travelling from $i$ to $j$. For detailed explanations see Ezzine and Elloumi (2012).

We now present some remarks and comments on this formulation in the next section.

\section{Comments on (EE) formulation}

First of all, there are some typos in the formulation. Summation in the constraints given in equations (EE2) and (EE3) are over all $i$ 's and thus $\forall(i=1,2, \ldots, n)$ part is wrong. 
Remark 1: The constraints given in equation (EE6) is not valid for k-TRP.

$x_{i j}+x_{j i}=1, \forall i, j \varepsilon A$ means, for each pair of fixed customers $i$ and $j$, either entering or leaving arc must be on the path. This type of assignment is actually assigning each pair to each other, which is definitely not necessarily true. With this form of the constraint (EE6) the model will be infeasible. The correct form of this constraint should be $x_{i j}+x_{j i} \leq 1, \forall i$, $j \varepsilon A$, then it will be valid for k-TRP.

Remark 2: Constraints given in equations (EE7) and (EE8) produces same set of inequalities. Thus only one of them may be taken into account. In addition, the index $m$ must also be different from $i$ and $j$

Example: Let $m=2, i=3, j=4$, then constraint (EE7) produces

$$
x_{32}+x_{24}+x_{43} \leq 2
$$

and constraint (EE8) produces:

$$
x_{34}+x_{42}+x_{23} \leq 2
$$

Secondly, by changing last two indices, let $m=2, i=4, j=3$, the constraint (EE7) produces:

$$
x_{42}+x_{23}+x_{34} \leq 2
$$

and constraint (EE8) produces:

$$
x_{43}+x_{32}+x_{24} \leq 2
$$

As it is easily seen, inequalities (1) and (4), and (2) and (3) are equivalent. Thus, for different values of $m, i$ and $j$, (EE7) and (EE8) produce same group of inequalities, which also show that one of them is redundant.

As many of the routing problems k-TRP, are among the variants of the well known travelling salesman problem (TSP). The following inequalities known as Danzing-Fulkerson-Johnson (DFJ) (Danzing et al., 1954; Öncan et al., 2009) sub tour elimination constraints for TSP. They are also valid inequalities for assignment-based formulations of the variants of TSP.

$$
\sum_{(i, j) \in S} x_{i j} \leq|S|-1, \quad \forall S \subset V,|S| \geq 2
$$

Remark 3: Corrected form of the constraint (EE6) is the DFJ for $|S|=2$ and constraints (EE7) and (EE8) are the DFJ for $|S|=3$. So, those constraints are not new. In addition, our computational experiments show that, if the formulation has another sub tour elimination constrains, as the formulation has (EE9), to incorporate the DFJ inequalities for $|S|=2$ and $|S|=3$ do not make significant improvement on the CPU time and/or linear programming relaxation of the model. Thus, there is no need for those constraints.

Remark 4: The constraint given in equation (EE13) is redundant due to equations (EE9) and (EE10).

$x_{i 1}$ is either ' 0 ' or ' 1 '. If $x_{i 1}=1$ then from equation (EE10) we get $y_{i 1}=1$. If the traveller arrives at the node $i$ from the node $k$, from equation (EE9), we get $y_{k i}=2$. Since the equation (EE9) adds 1 for each previous $y_{i j}$ 's values, the maximal value that a $y_{1 j}$ can take 
must be less than or equal to $n-k+1$. Therefore, we do not need additional constraint for upper bounds. Thus, constraint (EE13) is redundant.

Remark 5: We do not need to impose $y_{i j}$ 's to be integer. Those variables automatically take integer values since we have constraints (EE10) and (KKY7), and constraints (EE9) and (KKY6).

Remark 6: The authors cite the work of Kara et al. (2008, p.506) by saying that "This formulation is derived from an integer programming model introduced by Kara et al. (2008) for the CCVRPs in fact CumVRP), from which we only keep the assignment constraints." The assignment constraints are given in equations (KKY2)-(KKY5) and equations (EE2)-(EE5). In fact, in addition to assignment constraints except DFJ-based constraints given in equations (EE6), (EE7) and (EE8), and redundant constraints (EE13), which are discussed in Remarks 1, 2 and 3 above, the formulation (EE) is exactly the same as Kara et al. (2008) formulation proposed for k-TRP. A close look at both formulations, one can easily see that definitions of the decision variables, mathematical form of the objective functions [equations (KKY1) and (EE1)], sub tour elimination constraints (KKY6) and (EE9), bounding constraints (KKY7), (KKY8), (KKY9) and constrains (EE10), (EE11) and (EE12) are all same.

Remark 7: The authors claim that, with their words, "To the best of our knowledge, we are the first to propose a mathematical programming for the problem" (p.506). This statement is not correct. Kara et al. (2008) propose a general mixed integer linear programming model for cumulative vehicle routing problem. Later they show that the proposed formulation reduces to a formulation for k-TRP when the demand of each customer is taken as 1 . The reduced form of the EE is same as the formulation KKY given above. So, the first mathematical programming model for k-TRP was proposed by Kara et al. (2008). This fact is pointed out by Nucamendi-Guillen et al. (2016) also.

\section{Conclusions}

As explained and detailed in Section 3, EE formulation differs from Kara et al. (2008) with four group of constraints (EE6), (EE7), (EE8) and (EE13). We show that equation (EE6) is not valid; equations (EE7) and (EE8) are well known DFJ inequalities of routing models with subsets of 3 nodes, and equation (EE13) is redundant. We also showed that since there is equation (EE9), we do not need DFJ types sub tour elimination constraints. We conclude that, Ezzine and Elloumi (2012) formulation is not a new formulation for k-TRP, and thus Kara et al. (2008) formulation is the first polynomial size mathematical model for k-TRP. For different variations of k-TRP, we refer the researchers to Kara et al. (2017) where new formulations for k-TRP are presented.

\section{References}

Angel-Bello, F., Alvarez, A. and Garcia I. (2013) 'Two improvement formulations for the minimum latency problem', Applied Mathematical Modeling, Vol. 37, pp.2257-2266.

Danzing, G.B., Fulkerson, D.R. and Johnson, S.M. (1954) 'Solution of a large-scale traveling salesman problem’, Operations Research, Vol. 2, pp.393-410. 
Ezzine, I.O. and Elloumi, S. (2012) 'Polynomial formulation and heuristic based approach for k-travelling repairman problem', Int. J. Mathematics in Operational Research, Vol. 4, No. 5, pp.503-514.

Fischetti, M., Laporte G. and Martello S. (1993) 'The delivery man problem and cumulative matroids', Operations Research, Vol. 41, No. 6, pp.1055-1064.

Flores-Garza, D., Salazar-Aguilar, M., Ngueveu, S. and Laporte, G. (2017) 'The multi-vehicle cumulative covering tour problem', Annals of Operations Research, Vol. 258, No. 2, pp.761-780.

Jothi, R. and Raghavachari, B. (2007) 'Approximating the k-traveling repairman problem with repairtimes', Journal of Discrete Algorithms, Vol. 5, No. 1, pp.293-303.

Kara, İ., Kara, B.Y. and Yetis, M.K. (2008) 'Cumulative vehicle routing problems', in Caric, T. and Gold, H. (Eds.): Vehicle Routing Problem, Vol. 6, pp.85-98, In-Tech, Vienne, Austria.

Kara, İ., Önder, G. and Derya, T. (2017) 'Formulations for the multiple traveling repairman problem: computational analysis and some extensions', Presented at the IEOM2007, Rabat, Morocco, April, pp.11-13.

Nucamendi-Guillen, S., Martinez-Salazar, I., Angel-Bello, F. and Moreno-Vega J.M. (2016) 'A mixed integer formulation and an efficient metaheuristic procedure fort the k-travelling repairmen problem', Journal of the Operational Research Society, Vol. 67, No. 8, pp.1121-1134.

Öncan, T., Kuban, A.İ. and Laporte, G. (2009) 'A comparative analysis of several asymmetric traveling salesman problem formulations', Computers and Operations Research, Vol. 36, No. 3, pp.637-654.

Önder, G., Kara, İ. and Derya, T. (2016) 'New integer programming formulation for multiple traveling repairmen problem', 19th EURO Working Group on Transportation Meeting, EWGT2016, Istanbul, Turkey, 5-7 September.

Tsitsiklis, J.N. (1992) 'Special cases of traveling salesman and repairman problems with time windows', Networks, Vol. 22, pp.263-282. 